\title{
REVIEW
}

\section{Vitamin D endocrine system and osteoclasts}

\author{
Naoyuki Takahashi ${ }^{1}$, Naoyuki Udagawa ${ }^{2}$ and Tatsuo Suda ${ }^{3}$ \\ ${ }^{1}$ Institute for Oral Science, Matsumoto Dental University, Nagano, Japan. ${ }^{2}$ Department of Biochemistry, Matsumoto Dental \\ University, Nagano, Japan. ${ }^{3}$ Research Center for Genomic Medicine, Saitama Medical University, Saitama, Japan.
}

\begin{abstract}
Vitamin D was discovered as an anti-rachitic agent preventing a failure in bone mineralization, but it is now established that the active form of vitamin $D_{3}\left(1 \alpha, 25(\mathrm{OH})_{2} \mathrm{D}_{3}\right)$ induces bone resorption. Discovery of the receptor activator of nuclear factor $-\kappa B$ ligand (RANKL) uncovered the molecular mechanism by which $1 \alpha, 25(\mathrm{OH})_{2} \mathrm{D}_{3}$ stimulates bone resorption. Treating osteoblastic cells with $1 \alpha, 25(\mathrm{OH})_{2} \mathrm{D}_{3}$ stimulates RANKL expression, which in turn induces osteoclastogenesis. Nevertheless, active vitamin $D$ compounds such as calcitriol $\left(1 \alpha, 25(\mathrm{OH})_{2} \mathrm{D}_{3}\right)$, alfacalcidol $\left(1 \alpha(\mathrm{OH}) \mathrm{D}_{3}\right)$ and eldecalcitol (1 $\alpha, 25$-dihydroxy-2 $\beta$-(3-hydroxypropoxy) vitamin $D_{3}$ ) have been used as therapeutic drugs for osteoporosis, as they increase bone mineral density (BMD) in osteoporotic patients. Paradoxically, the increase in BMD is caused by the suppression of bone resorption. Several studies have been performed to elucidate the mechanism by which active vitamin D compounds suppress bone resorption in vivo. Our study showed that daily administration of eldecalcitol to mice suppressed neither the number of osteoclast precursors in the bone marrow nor the number of osteoclasts formed in ex vivo cultures. Eldecalcitol administration suppressed RANKL expression in osteoblasts. This review discusses how the difference between in vitro and in vivo effects of active vitamin $D$ compounds on bone resorption is induced.
\end{abstract}

BoneKEy Reports 3, Article number: 495 (2014) | doi:10.1038/bonekey.2013.229

\section{Introduction}

Vitamin D was discovered as an anti-rachitic agent capable of preventing a failure in bone mineralization. A vitamin D deficiency results in rickets in the young and osteomalacia in adults. The administration of vitamin $\mathrm{D}$ to rachitic animals and humans cures impaired bone mineralization. Although vitamin $D$ is the sole factor which stimulates intestinal absorption of calcium, vitamin $\mathrm{D}$ and parathyroid hormone (PTH) working in concert are necessary to mobilize calcium from the bone and conserve calcium from urine (Figure 1). Therefore, vitamin $D$ is postulated to directly stimulate osteoblastic bone formation and mineralization; however, there is no evidence to support this proposal.

Vitamin $D_{3}$ is first metabolized to 25-hydroxyvitamin $D_{3}$ $\left(25(\mathrm{OH}) \mathrm{D}_{3}\right)$ in the liver and then to $1 \alpha, 25$-dihydroxyvitamin $\mathrm{D}_{3}$ $\left(1 \alpha, 25(\mathrm{OH})_{2} \mathrm{D}_{3}\right)$ in the kidney. ${ }^{1,2} 1 \alpha, 25(\mathrm{OH})_{2} \mathrm{D}_{3}$ is now recognized as a steroid hormone that has a role in maintaining calcium homeostasis through the vitamin $\mathrm{D}$ receptor (VDR). ${ }^{3}$ VDR knockout $\left(\mathrm{VDR}^{-1-}\right)$ mice grew normally before weaning. ${ }^{4}$ However, they developed severe hypocalcemia after weaning. Both bone formation and mineralization were severely impaired in $\mathrm{VDR}^{-1-}$ mice but were completely recovered by feeding a high-calcium diet. ${ }^{4}$ When the bone isolated from $\mathrm{VDR}^{-1-}$ mice was transplanted into wild-type (WT) mice, the $\mathrm{VDR}^{-1-}$ bone showed excessive bone formation and mineralization under normocalcemic conditions. ${ }^{5}$ These results suggest that the stimulating effect of $1 \alpha, 25(\mathrm{OH})_{2} \mathrm{D}_{3}$ on bone mineralization is indirect, occurring through stimulation of intestinal calcium absorption.

Carlsson $^{6}$ and Bauer ${ }^{7}$ were the first to realize that a major function of vitamin $\mathrm{D}$ in the bone was to induce bone resorption. Using an organ culture system, Raisz et al. ${ }^{8}$ showed that $1 \alpha, 25(\mathrm{OH})_{2} \mathrm{D}_{3}$ increased the release of ${ }^{45} \mathrm{Ca}$ from the prelabeled bone into the culture medium. When the bone marrow cells were cultured, multinucleated cells having the characteristics of osteoclasts were formed in response to bone-resorbing factors, including $1 \alpha, 25(\mathrm{OH})_{2} \mathrm{D}_{3} \cdot{ }^{9-11}$ These results established the concept that $1 \alpha, 25(\mathrm{OH})_{2} \mathrm{D}_{3}$ was an inducer of osteoclastic bone resorption.

Nevertheless, active vitamin $\mathrm{D}$ compounds such as calcitriol $\left(1 \alpha, 25(\mathrm{OH})_{2} \mathrm{D}_{3}\right)$, alfacalcidol (1 $\alpha$-hydroxyvitamin $\left.\mathrm{D}_{3}, 1 \alpha(\mathrm{OH}) \mathrm{D}_{3}\right)$

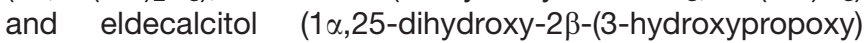
vitamin $D_{3}$ ) have been used as therapeutic drugs to treat osteoporosis in Japan, because they improve bone mineral density (BMD) and decrease the risk of fractures. ${ }^{12-16}$ Paradoxically, such beneficial effects are caused by inhibiting osteoclastogenesis. The present review describes the regulation of osteoclast differentiation and possible mechanisms by which active vitamin $\mathrm{D}$ compounds suppress bone resorption in vivo.

Correspondence: Professor N Takahashi, Institute for Oral Science, Matsumoto Dental University, 1780 Hiro-oka Gobara, Shiojiri, Nagano 399-0781, Japan. E-mail: takahashinao@po.mdu.ac.jp 


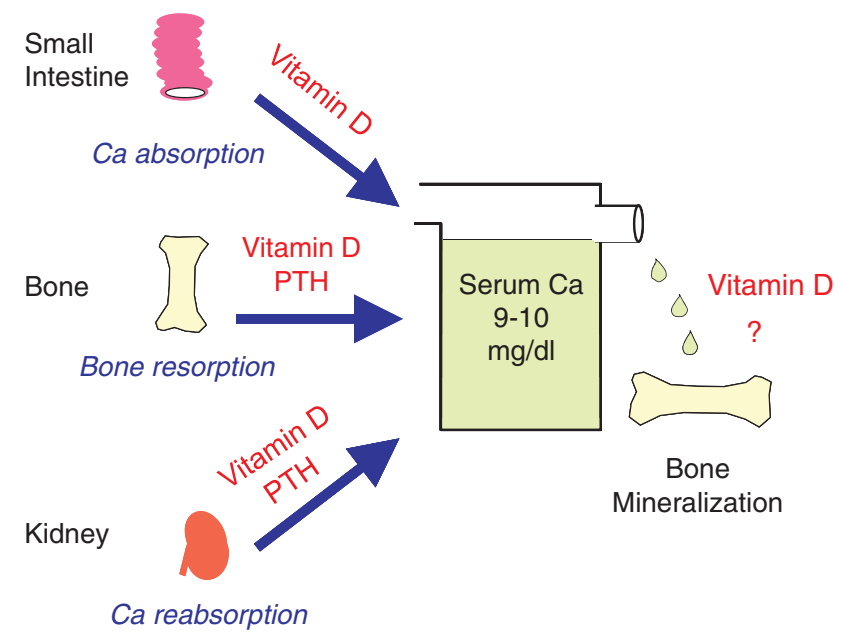

Figure 1 Classical actions of vitamin D to maintain serum calcium homeostasis. Vitamin $D$ is the sole factor that stimulates intestinal calcium absorption. It is proposed that vitamin D and PTH working in concert are necessary to mobilize calcium from the bone and conserve calcium from urine. However, there is no direct evidence that vitamin $\mathrm{D}$ directly stimulates osteoblastic bone mineralization.

\section{Structure and Function of Osteoclasts}

Osteoclasts have several characteristics, including multiple nuclei, abundant mitochondria and a large number of vacuoles and lysosomes (Figure 2). ${ }^{17,18}$ The most characteristic feature of bone-resorbing osteoclasts is the presence of ruffled borders and sealing zones (also called clear zones). The sealing zone attaches osteoclasts to the bone surface and isolates resorption lacunae from the surroundings. Resorption lacunae under the ruffled border are acidic, which favors the dissolution of bone minerals. Vacuolar $\mathrm{H}^{+}$-ATPase is localized in the ruffled border membranes. The transport of protons into resorption lacunae is mediated by vacuolar $\mathrm{H}^{+}$-ATPase. Lysosomal enzymes are also secreted into lacunae to degrade the organic matrix in the bone. Tartrate resistant-acid phosphatase (TRAP) is highly expressed in osteoclasts, and some TRAP is secreted into resorption lacunae. Histochemical TRAP staining is widely used to identify osteoclasts in vivo and in vitro. Some matrix degradation products are endocytosed from the central portion of the ruffled border domain, incorporated into transcytotic vesicles and exocytosed through the functional secretory domain in the basolateral membrane. ${ }^{18}$ Osteoclasts express calcitonin receptors. Calcitonin suppresses bone-resorbing activity of osteoclasts. Osteoclasts also express the vitronectin receptor, $\alpha_{v} \beta_{3}$ integrin, which is involved in attachment to the bone matrix. Recently, Fuller et al. ${ }^{19}$ reported that $\alpha_{v} \beta_{3}$ integrins were not only necessary but also sufficient for the induction of resorptive behavior in osteoclasts.

Multinucleated osteoclasts are formed by cell-cell fusion of mononuclear preosteoclasts. The dendritic cell-specific transmembrane protein (DC-STAMP), a seven-transmembrane protein, was first identified as a protein responsible for the fusion of preosteoclasts. ${ }^{20,21}$ No multinucleated osteoclasts were observed, but many preosteoclasts were detected in DC-STAMP ${ }^{-1-}$ mice. The bone-resorbing activity of DCSTAMP $^{-1-}$ preosteoclasts was lower than that in multinucleated osteoclasts. DC-STAMP ${ }^{-1-}$ mice develop mild osteopetrosis. Miyamoto et al. ${ }^{22}$ recently reported that

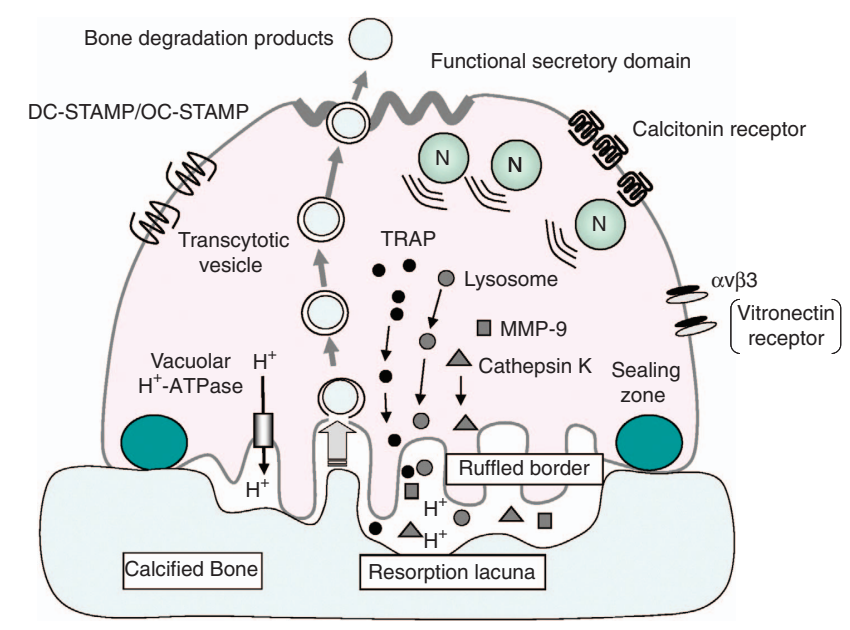

Figure 2 Ultrastructure and function of osteoclasts. Osteoclasts have several characteristics, such as multiple nuclei, abundant mitochondria and a large number of vacuoles and lysosomes. Bone-resorbing osteoclasts form ruffled borders and sealing zones. The resorbing area under the ruffled border is acidic. Vacuolar $\mathrm{H}^{+}$-ATPase localized in the ruffled border is involved in the transport of protons into the resorption lacunae. Enzymes such as cathepsin K, MMP9 and TRAP are secreted into the resorption lacuna to degrade bone matrix proteins. Matrix degradation products are endocytosed from the central portion of the ruffled border, packaged into transcytotic vesicles and secreted from the functional secretory domain. Osteoclasts express large numbers of calcitonin receptors and $\alpha_{v} \beta_{3}$ vitronectin receptors. Osteoclasts also express DC-STAMP and OC-STAMP, which are involved in the cell-cell fusion of osteoclasts.

osteoclast-stimulatory transmembrane protein (OC-STAMP), another seven-transmembrane protein, was also involved in the fusion of preosteoclasts. OC-STAMP ${ }^{-1-}$ mice exhibited a complete lack of cell-cell fusion of preosteoclasts, although the expression of DC-STAMP was normal in these cells. These results suggest that the fusion of osteoclasts is regulated by both OC-STAMP and DC-STAMP.

\section{Regulation of Osteoclast Differentiation by Osteoblastic Cells}

In 1981, Rodan and Martin ${ }^{23}$ proposed that osteoblasts may intervene in the process of osteoclastic bone resorption. Their argument was based on observations that osteoblasts, but not osteoclasts, expressed receptors of bone-resorbing factors, such as PTH and prostaglandin $\mathrm{E}_{2}\left(\mathrm{PGE}_{2}\right)$. Bone-lining cells were also suggested to be involved in the regulation of osteoclastic bone resorption. ${ }^{24}$ Based on this concept, we established a mouse co-culture system of calvarial osteoblastic cells and splenocytes to investigate osteoclastogenesis. Osteoclast-like multinucleated cells were formed in this coculture in response to bone-resorbing factors, including $1 \alpha, 25(\mathrm{OH})_{2} \mathrm{D}_{3}, \mathrm{PTH}$ and $\mathrm{PGE}_{2} .{ }^{25}$ No osteoclasts were formed when cell-to-cell contact between osteoblastic cells and splenocytes was inhibited by a membrane filter. These results suggest that microenvironments provided by osteoblastic cells support the osteoclastic differentiation of splenic precursors.

Experiments with the osteopetrotic op/op mice have established the role of macrophage colony-stimulating factor (M-CSF) for osteoclastogenesis. An extra thymidine insertion in the M-CSF gene was found in op/op mice, which generated a stop codon in the downstream, ${ }^{26}$ suggesting that op/op mice could not produce the active M-CSF protein. The administration 
of recombinant M-CSF to op/op mice restored impaired bone resorption. ${ }^{27}$ Osteoblastic cells obtained from op/op mice could not support osteoclastogenesis in co-cultures with WT splenocytes. ${ }^{28}$ The addition of M-CSF to the co-culture with op/ op osteoblastic cells induced osteoclast formation from WT splenocytes in response to $1 \alpha, 25(\mathrm{OH})_{2} \mathrm{D}_{3}$. In contrast, op/op splenic precursors differentiated into osteoclasts when cocultured with WT osteoblastic cells. M-CSF was shown to be involved not only in the proliferation of osteoclast precursors but also in their differentiation into osteoclasts. ${ }^{29}$

In 1992, we proposed a hypothesis for the mechanism of osteoclastogenesis: bone-resorbing factors act on osteoblastic cells to induce a membrane-bound factor, named 'osteoclast differentiation factor (ODF)'. ${ }^{30}$ Osteoclast precursors express ODF receptors, recognize ODF through cell-cell interaction with osteoblastic cells and differentiate into osteoclasts in the presence of M-CSF. Chambers et al. ${ }^{31}$ reported a similar factor 'stromal cell-derived osteoclast forming activity'.

\section{Discovery of the RANKL-RANK Interaction for Osteoclastogenesis}

Osteoprotegerin (OPG; also called osteoclastogenesis inhibitory factor (OCIF)) was cloned as a member of the tumour necrosis factor (TNF) receptor family in $1997 .^{32,33}$ This protein lacked a transmembrane domain but had an N-terminal signal peptide for a secreted protein. OPG/OCIF suppressed osteoclast formation in co-cultures treated with bone-resorbing factors. $^{32}$ Therefore, OPG/OCIF was speculated to act as a decoy receptor for ODF. Using OPG/OCIF as a probe, the ODF cDNA was isolated from a library of the bone marrow stromal cell line ST2. ${ }^{34}$ ODF is a transmembrane protein of the TNF ligand family, and its expression in osteoblastic cells was upregulated by bone-resorbing factors. Lacey et al. ${ }^{35}$ also cloned an OPG ligand (OPGL), which was identical to ODF. Molecular cloning of ODF/OPGL demonstrated that ODF/OPGL was identical to TNF-related activation-induced cytokine $\left(\right.$ TRANCE) ${ }^{36}$ and receptor activator of nuclear factor (NF)- $\mathrm{B} B$ ligand (RANKL), ${ }^{37}$ which had been identified by other groups. The receptor of ODF was confirmed to be receptor activator of NF- $\kappa B$ (RANK). Thus, ODF, OPGL, TRANCE and RANKL are different names for the same ligand, and OPG and OCIF are different names for the same decoy receptor. ${ }^{38,39}$ According to the President's Committee on Nomenclature (2000) of the American Society for Bone and Mineral Research, the terms 'RANKL' as the ligand, 'RANK' as the receptor and 'OPG' as the decoy receptor are used in text (Figure 3).

Osteoblastic cells express RANKL as a membraneassociated factor in response to bone-resorbing factors. ${ }^{38}$ $1 \alpha, 25(\mathrm{OH})_{2} \mathrm{D}_{3}$ is one of the most potent inducers of RANKL in osteoblastic cells. VDR ${ }^{-1-}$ osteoblastic cells failed to support $1 \alpha, 25(\mathrm{OH})_{2} \mathrm{D}_{3}$-induced osteoclastogenesis in co-cultures with WT splenocytes. ${ }^{40}$ The molecular mechanism by which $1 \alpha, 25(\mathrm{OH})_{2} \mathrm{D}_{3}$ enhances transcription of the RANKL gene is described by Pike et al. ${ }^{41}$

\section{NFATc1 as a Master Transcription Factor for Osteoclastogenesis}

Signaling pathways required for osteoclastogenesis have been identified since the discovery of RANKL (Figure 4). ${ }^{40,42-44}$

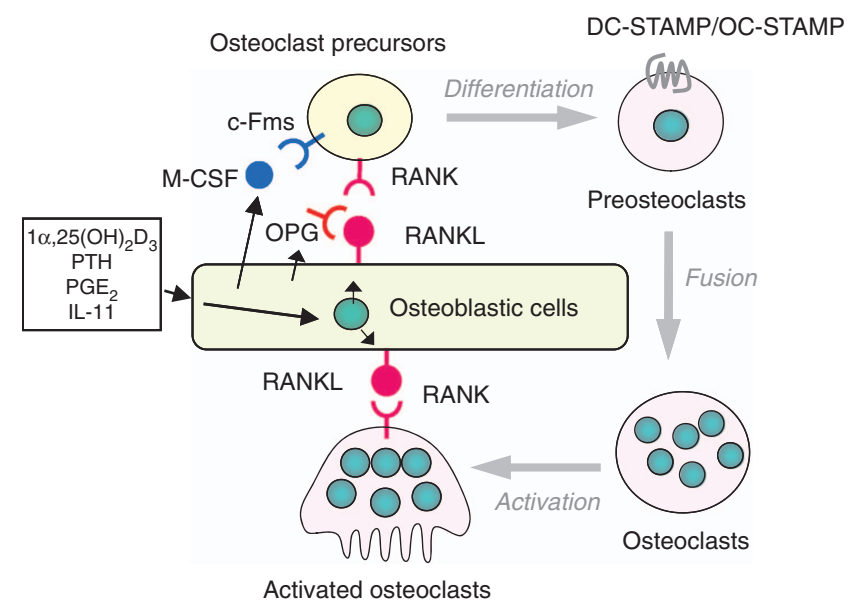

Figure 3 Regulation of osteoclast differentiation and function by osteoblastic cells. Bone resorption-stimulating factors act on osteoblastic cells to induce the expression of RANKL as a membrane-associated factor. Osteoblastic cells constitutively produce M-CSF. Osteoclast precursors express receptors RANK and c-Fms and differentiate into osteoclasts in the presence of RANKL and M-CSF. Osteoblastic cells secrete OPG, which inhibits the RANKL-RANK interaction between osteoblastic cells and osteoclast precursors. Multinucleated osteoclasts also express RANK, and RANKL induces the bone-resorbing activity of osteoclasts via the interaction with RANK.

Binding of RANKL to RANK triggers TNF receptor-associated factor 6 (TRAF6)-dependent signaling, which activates phospholipase $\mathrm{C} \gamma$ (PLC $\gamma$ ), mitogen-activated protein (MAP) kinases and NF- $\mathrm{KB}$. RANKL-induced osteoclastogenesis is also dependent on co-stimulatory signaling through immunoreceptor tyrosine-based activation motif (ITAM)-containing adaptors, Fc receptor common $\gamma(\mathrm{FcR} \gamma)$ and DNAX-activating protein of $12 \mathrm{kDa}$ (DAP12). ${ }^{45} \mathrm{FcR} \gamma$ associates with osteoclast-associated receptor (OSCAR), while DAP12 associates with triggering receptor expressed on myeloid cells 2 (TREM-2). RANKmediated and ITAM-mediated signals cooperate to further activate PLC $\gamma$, which generates inositol-1,4,5-triphosphate $\left(\mathrm{IP}_{3}\right)$. $\mathrm{IP}_{3}$ then induces $\mathrm{Ca}^{2+}$ mobilization from the endoplasmic reticulum through $\mathrm{IP}_{3}$ receptors and generates $\mathrm{Ca}^{2+}$ oscillations. $\mathrm{Ca}^{2+}$ oscillations contribute to the amplification of NF of activated T-cells, cytoplasmic 1 (NFATc1), the master transcription factor for osteoclastogenesis. ${ }^{44}$ Because ITAMmediated signaling is crucial for the robust induction of NFATc1, this pathway is called 'co-stimulatory signaling' for RANKinduced osteoclastogenesis. Osteoblastic cells have been proposed to express ligands for OSCAR and TREM-2. OSCAR was recently shown to bind to a specific motif of collagen. ${ }^{46}$

NFATc1 is also activated by a $\mathrm{Ca}^{2+}$ oscillation-independent pathway (Figure 3). ${ }^{47}$ This pathway, as well as the ITAM pathway, is activated by osteobalstic cells. FK506, an inhibitor of calcineurin, suppresses $\mathrm{Ca}^{2+}$ oscillations in osteoclast precursors. NFATc1 concentrations in osteoclast precursors were increased in co-cultures with osteoblastic cells even in the presence of FK506. Osteoclast precursors derived from $\mathrm{IP}_{3}$ receptors type 2 and type 3 double knockout mice, in which RANKL-induced $\mathrm{Ca}^{2+}$ oscillations were absent, normally differentiated into osteoclasts in co-culture with WT osteoblastic cells. ${ }^{47}$ Cot (cancer osaka thyroid) serine/threonine kinase in osteoclast precursors was activated by cell-cell interactions with osteoblastic cells. ${ }^{48}$ The activation of Cot in osteoclast precursors increased NFATc1 protein levels through 


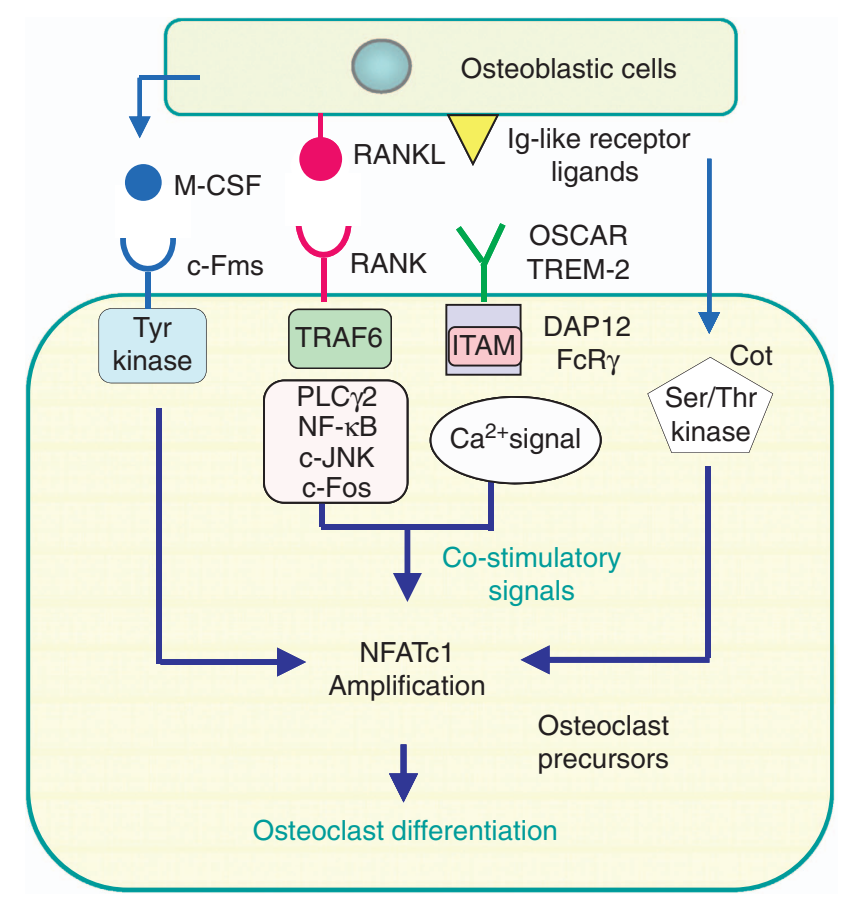

Figure 4 Intracellular signals in osteoclast precursors induced by osteoblastic cells. Osteoclast differentiation is induced by amplifying NFATc1, a master transcription factor for osteoclastogenesis. The M-CSF receptor, c-Fms, has a tyrosine kinase domain in the cytoplasmic region, and tyrosine kinase-mediated signals regulate the proliferation and differentiation of osteoclast precursors. The binding of RANKL to RANK results in the recruitment of TRAF6, which activates PLC $\gamma$, MAP kinases, NF- $\mathrm{kB}$ and AP1 (c-Fos/ c-Jun). Immunoglobulin-like receptors, TREM2 and OSCAR, are associated with ITAMcontaining DAP12 and FcR $\gamma$, respectively. Osteoblastic cells express the ligands of immunoglobulin-like receptors. RANK and ITAM signaling lead to $\mathrm{Ca}^{2+}$ oscillations, which induce the amplification of NFATc1. NFATc1 is also activated in a $\mathrm{Ca}^{2+}$ oscillation-independent, but Cot kinase-dependent, manner. Cot is activated by the cell-cell interaction with osteoblastic cells.

phosphorylation-dependent protein stabilization. These results suggest that NFATc1 amplification is induced by both $\mathrm{Ca}^{2+}$ oscillation-dependent and -independent pathways. Mice doubly deficient in DAP12 and FcR $\gamma$ have been shown to exhibit severe osteopetrosis owing to the lack of osteoclasts. ${ }^{45}$ These results suggest that the ITAM signal is physiologically important in controlling osteoclast differentiation.

\section{Characteristics of Osteoclast Precursors In Vivo}

Attempts to identify osteoclast precursors in vivo have established a model for osteoclastogenesis. Mizoguchi et al. ${ }^{49}$ reported that cell cycle progression and subsequent cell cycle arrest in osteoclast progenitors were required for their differentiation into direct osteoclast precursors (Figure 5). The expression of cyclins and cyclin-dependent kinases (Cdks) was suppressed, whereas that of $\mathrm{p} 27^{\mathrm{KIP} 1}$, a Cdk inhibitor, was upregulated in the precursors during their differentiation into osteoclasts. ${ }^{49}$ Neither these precursors nor osteoclasts expressed Ki67, a cell proliferation marker. Therefore, these osteoclast precursors were named 'cell cycle-arrested quiescent osteoclast precursors' (QOPs). QOPs, but not osteoclasts, exist in RANKL ${ }^{-1-}$ mice and op/op mice. Bromodeoxyuridine (BrdU) is a nucleoside analog that is incorporated into dividing nuclei. $\mathrm{RANKL}^{-1-}$ mice were given

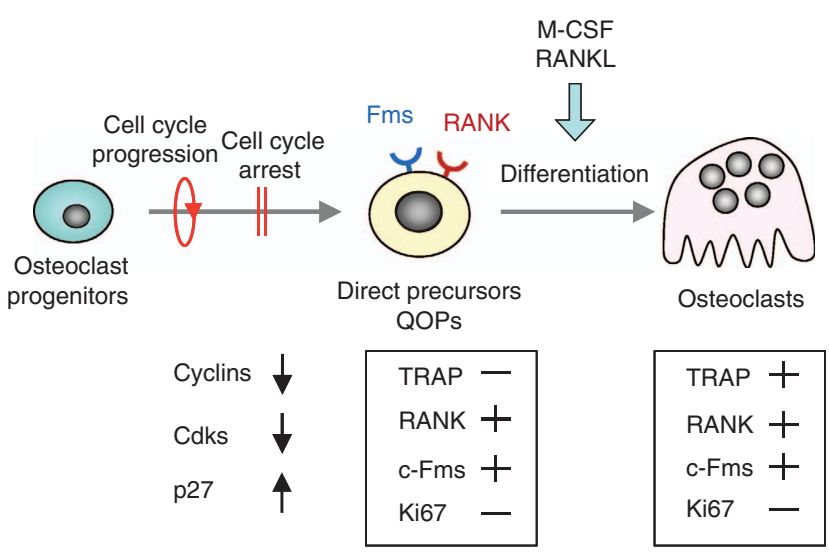

Figure 5 Two cell cycle-related events in osteoclastogenesis. Cell cycle progression and subsequent withdrawal in osteoclast progenitors are required for their differentiation into osteoclasts in vitro. The direct osteoclast precursors have been named 'cell cycle-arrested quiescent osteoclast precursors (QOP)'. The cell cycle arrest in QOP is induced by the disappearance of cyclins and Cdks, and the appearance of p27 ${ }^{\mathrm{KIP} 1}$. Osteoclasts express TRAP, RANK and c-Fms, but not Ki67, while QOP express RANK and c-Fms but not TRAP or Ki67.

BrdU in their drinking water and were injected with RANKL for 2 days. Osteoclasts appeared in the bone of $\mathrm{RANKL}^{-1-}$ mice in response to the RANKL injection. More than $70 \%$ of nuclei in RANKL-induced osteoclasts were negative for BrdU. ${ }^{49}$ op/op mice were also given BrdU and were injected with M-CSF for 7 days. Many osteoclasts appeared in the bone in op/op mice injected with M-CSF. More than $80 \%$ of nuclei in those osteoclasts were BrdU negative. ${ }^{49}$ These results suggest that osteoclasts are formed from QOPs in both RANKL ${ }^{-1-}$ mice and op/op mice.

QOPs are expected to express RANK and c-Fms, but not TRAP or Ki67. The distribution of QOPs was examined in RANKL $^{-1-}$ mice using these markers, because QOPs, but not osteoclasts, exist in these mice. RANK and c-Fms-double positive $\left(\mathrm{Fms}^{+} / \mathrm{RANK}^{+}\right)$cells were detected along the surface of trabecular bones in RANKL ${ }^{-1-}$ mice. ${ }^{49}$ They were negative for Ki67. QOPs were detected near alkaline phosphatasepositive $\left(\mathrm{ALP}^{+}\right)$osteoblasts, suggesting that osteoblasts support the presence of QOPs in the bone.

QOPs were isolated as RANK-positive $\left(\mathrm{RANK}^{+}\right)$cells from the WT bone marrow. RANK ${ }^{+}$cells expressed c-Fms but not macrophage-associated markers, such as F4/80 and CD11b. Bone marrow-derived QOPs showed no phagocytic activity and did not proliferate in response to M-CSF. ${ }^{50}$ They could not differentiate into dendritic cells but differentiated into osteoclasts. These results suggest that QOPs are committed precursors of osteoclasts.

\section{Circulating Osteoclast Precursors}

Some QOPs are circulating. Collagen disks containing bone morphogenetic protein 2 (BMP-2) were implanted into WT mice. These mice were given BrdU. After implantation for 2 weeks, osteoclasts were detected in ectopic bone tissues induced by BMP-2. Most nuclei in these osteoclasts were BrdU negative. ${ }^{50}$ Osteoclasts were not induced in control collagen disks. Collagen disks containing BMP-2 were also implanted into $\mathrm{RANKL}^{-1-}$ mice. QOPs appeared in BMP-2-induced bone 
tissues but not in the control disks in $\mathrm{RANKL}^{-/-}$mice. When RANKL was injected into these RANKL ${ }^{-/}$mice, osteoclasts appeared in BMP-2-containing disks but not in the control disk. ${ }^{51}$ These results suggested that some QOPs circulated in the blood and settled in the bone (Figure 6a). The distribution of QOPs was similar to that of $\mathrm{ALP}^{+}$osteoblasts in $\mathrm{RANKL}^{-1-}$ mice, ${ }^{49}$ suggesting that $\mathrm{ALP}^{+}$bone-forming osteoblasts are somehow involved in homing of QOPs to the bone. Using fluorescent imaging techniques, Kotani et al. ${ }^{52}$ showed that some osteoclasts were generated from circulating precursors. At present, it is not known that all QOPs found on the bone surfaces are derived from circulating QOPs.

Recent studies suggest that c-Fms-mediated signaling is required for the differentiation of hematopoietic progenitors into QOPs. Interleukin 34 (IL-34) is a newly discovered ligand for c-Fms. ${ }^{53}$ The amino-acid sequence of IL-34 is different from that of M-CSF; however, the biological activity of the two ligands is similar. IL-34 is predominantly expressed in the spleen and not in bone, while M-CSF is expressed in both the bone and spleen. c-Fms ${ }^{+} / \mathrm{RANK}^{+}$QOPs were not detected in the bone but were detected in the spleen of op/op mice. ${ }^{54}$ This suggested that QOPs moved from the spleen to the bone in response to $\mathrm{M}-\mathrm{CSF}$ injections. Indeed, removal of the spleen (splenectomy) blocked M-CSF-induced osteoclastogenesis in op/op mice. The expression of RANK in precursors is shown to be induced by M-CSF. ${ }^{55,56}$ These results suggest that IL-34 has a pivotal role in maintaining splenic QOPs in op/op mice and that some QOPs circulate in the blood. On the basis of these findings, we proposed a model of osteoclast formation in vivo (Figure 6a).
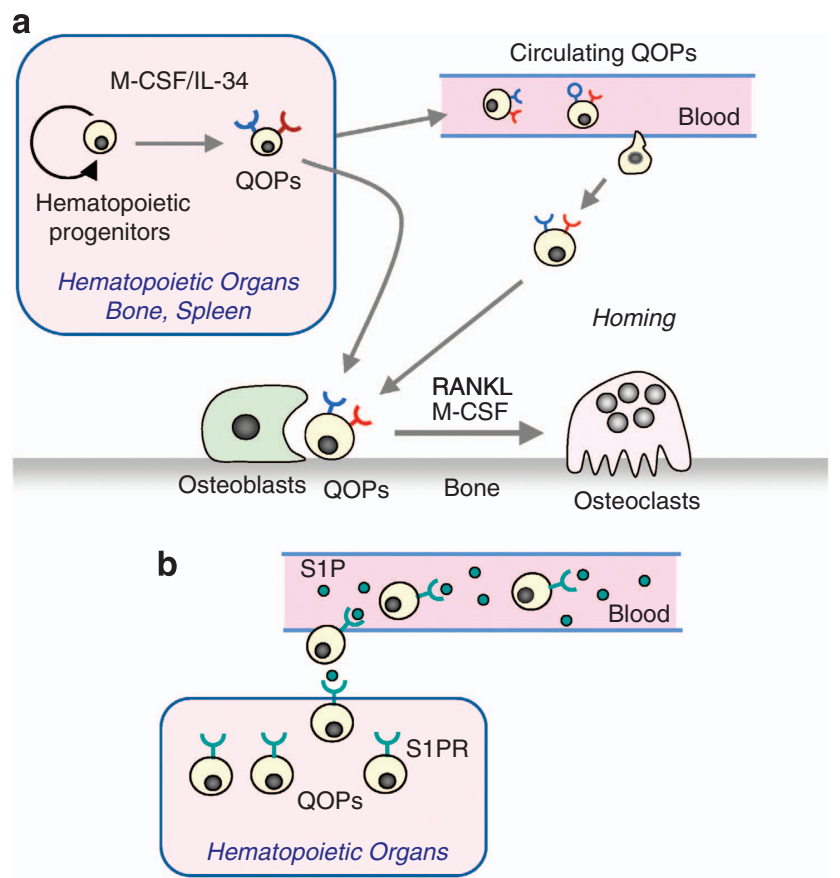

Figure 6 A hypothetical model for osteoclastogenesis in vivo. (a) QOPs are generated from hematopoietic progenitors in hematopoietic organs, such as the bone marrow and spleen. M-CSF and/or IL-34 are involved in the generation of QOPs. Some QOPs circulate in the blood. Osteoblasts may have a role in homing of QOPs to the bone. (b) S1P in the blood controls the trafficking of QOPs from hematopoietic tissues to the blood through S1P receptors (S1PR). Administration of FYT720, an S1P agonist, promotes the egress of QOPs from hematopoietic tissues into bloodstream.
M-CSF/IL-34 may be required for the differentiation of hematopoietic progenitors into QOPs. Some QOPs circulate in the blood and settle in the bone. Osteoblasts appear to be involved in homing of QOPs to the bone.

Sphingosine-1-phosphate (S1P), a lipid mediator enriched in the blood, was shown to control the dynamic migration of osteoclast precursors between the blood and bone. Ishii et al. ${ }^{57}$ reported that FYT720, an agonist of S1P, increased circulating osteoclast precursors and regulates bone homeostasis. We also confirmed that the injection of FTY720 to mice increased circulating QOPs in the blood. ${ }^{50}$ These results suggest that the interaction between $\mathrm{S} 1 \mathrm{P}$ and $\mathrm{S} 1 \mathrm{P}$ receptors $(\mathrm{SIPR})$ is involved in the egress of QOPs from hematopoietic tissues into the blood (Figure 6b).

\section{Effects of the In Vivo Administration of Vitamin D Compounds on Bone Metabolism}

Calcitriol, alfacalcidol and eldecalcitol have been used as therapeutic drugs for the treatment of osteoporosis in Japan. Alfacalcidol is rapidly converted to calcitriol by liver CYP27A1 (Figure 7a). Therefore, alfacalcidol is believed to act as calcitriol in vivo. Eldecalcitol was isolated from vitamin $D$ analogs based on the activity that stimulated $\mathrm{BMD}$ in vivo ${ }^{58}$ (Figure $7 \mathrm{~b}$ ). A clinical study showed that eldecalcitol increased BMD more effectively than alfacalcidol. ${ }^{16}$ Eldecalcitol binds more weakly to VDR but more strongly to serum vitamin D binding protein (DBP) than calcitriol. ${ }^{59}$ Eldecalcitol more weakly suppresses PTH production than calcitriol. The half-life of eldecalcitol in serum is
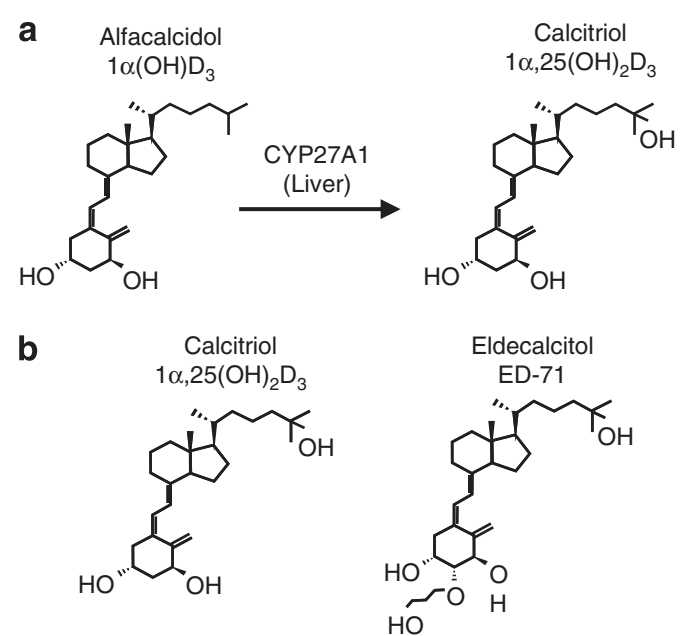

\begin{tabular}{lcc}
\hline & Calcitriol & Eldecalcitol \\
\hline Binding to VDR & 100 & 45 \\
Binding to DBP & 100 & 422 \\
PTH suppression & 100 & 3.5 \\
Half life in serum & Short & Long \\
\hline
\end{tabular}

Figure 7 Structures and characteristics of vitamin D compounds used as therapeutic drugs for osteoporosis. (a) Alfacalcidol, a prodrug of calcitriol $\left(1 \alpha, 25(\mathrm{OH})_{2} \mathrm{D}_{3}\right)$, is metabolized to calcitriol by liver CYP27A1. Alfacalcidol, therefore, acts as calcitriol in vivo. (b) Eldecalcitol has a hydroxypropoxy substituent at the $2 \beta$ position. Eldecalcitol is not metabolized to calcitriol in vivo. Some characteristics of eldecalcitol are compared with those of calcitriol. 
longer that than that of calcitriol. ${ }^{60}$ Such differences may be related to the higher efficacy of eldecalcitol than alfacalcidol.

The active form of vitamin $D$ enhances intestinal calcium absorption, leading to an increase in serum calcium levels and suppression of bone resorption. $1 \alpha, 25(\mathrm{OH})_{2} \mathrm{D}_{3}$ at pharmacological concentrations may also inhibit PTH secretion and stimulate calcium renal calcium re-absorption. Integrating such significant changes may suppress osteoclastic bone resorption. Recent data also indicate that autocrine/paracrine activities of vitamin $\mathrm{D}$ are detected in each of the major bone cell types where CYP27B1 $\left[25(\mathrm{OH}) \mathrm{D}_{3} 1 \alpha\right.$-hyrdoxylase] is expressed. ${ }^{61}$ Therefore, it is likely that $1 \alpha, 25(\mathrm{OH})_{2} \mathrm{D}_{3}$ produced by bone cells is also involved in the suppression of bone resorption in an autocrine/paracrine manner.

Clinical studies have shown that daily administration of eldecalcitol increases lumbar and hip BMD in osteoporotic patients with vitamin D supplementation. ${ }^{14,16}$ Transnet hypercalcemia was observed in some patients, but none of them sustained hypercalcemia. Both bone resorption and formation were suppressed by the administration of eldecalcitol, suggesting the existence of a coupling mechanism between bone resorption and bone formation. We therefore speculated that daily administration of active vitamin D compounds may directly suppress bone resorption.

We proposed two possible explanations for the inhibitory action of active vitamin $\mathrm{D}$ compounds on bone resorption in vivo. First, long-term exposure to pharmacological concentrations of active vitamin D compounds may alter the number of osteoclast precursors, including QOPs. The second possibility is that daily administration may alter the microenvironment of the bone, which supports osteoclastogenesis. We then examined the effects of the daily administration of eldecalcitol on osteoclastogenesis in mice ${ }^{62}$ (Figure 8). Treating mice with eldecalcitol increased BMD in the metaphysis of femurs. Bone and serum analyses showed that both bone resorption and formation were suppressed by eldecalcitol administration, suggesting that the eldecalcitol-induced increase in BMD was due to the suppression of bone resorption. ${ }^{62}$

We then examined how eldecalcitol suppressed bone resorption in vivo ${ }^{62}$ (Figure 8). Eldecalcitol administration failed to affect the number of QOPs in the bone marrow. F4/80 ${ }^{+}$and $\mathrm{CD}_{11} \mathrm{~b}^{+}$cells in the bone marrow are believed to be precursors of QOPs. The populations of $\mathrm{F} 4 / 80^{+}$and $\mathrm{CD} 11 \mathrm{~b}^{+}$cells in the bone marrow remained unchanged under the eldecalcitoltreated condition. An ex vivo culture showed that osteoclast formation from QOPs was not affected by eldecalcitol administration. We examined the second possibility that eldecalcitol may alter the microenvironment for osteoclastogenesis (Figure 8). Eldecalcitol administration significantly suppressed RANKL mRNA expression but not M-CSF or OPG mRNA expression in tibiae. RANKL ${ }^{+}$cells were immunohistochemically observed as a line in the hypertrophic cartilage area and around trabecular and cortical bones. The distribution of RANKL ${ }^{+}$cells was evaluated as RANKL-positive cell surface (RANKLS). Eldecalcitol administration significantly decreased RANKLS preferentially around trabecular bones. ${ }^{62}$ Bone loss in ovariectomized mice is an animal model of postmenopausal osteoporosis. The daily administration of eldecalcitol as well as calcitriol to ovariectomized mice suppressed RANK L expression in the bone and increased BMD. Eldecalcitol more

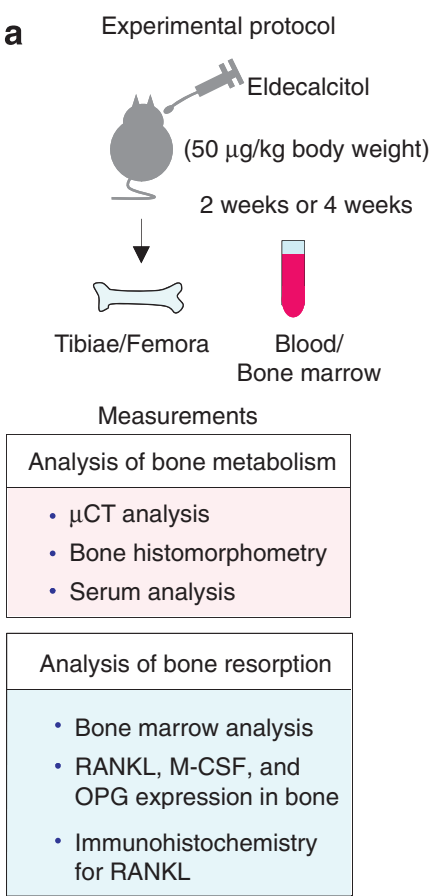

b

Effect of Eldecalcitol

\begin{tabular}{|l|}
\hline Analysis of bone metabolism \\
\hline Bone mass \\
\hline Bone resorption \\
\hline Bone formation \\
\hline Serum ALP \\
\hline Serum TRAP5b \\
\hline
\end{tabular}

\begin{tabular}{|l|}
\hline Analysis of bone resorption \\
\hline Number of QOPs \\
\hline $\begin{array}{l}\text { Ex vivo cultures } \\
\text { for osteoclastogenesis }\end{array}$ \\
\hline RANKL expression \\
\hline M-CSF expression \\
\hline OPG expression \\
\hline
\end{tabular}

Figure 8 Effects of the daily administration of eldecalcitol on bone metabolism in mice. (a) Protocol of experiments. Eldecalcitol ( $50 \mathrm{ng}$ per kg body weight) or vehicle was administered daily to 9 -week-old male mice for 2 and 4 weeks. Bone samples and serum were recovered for analyses of bone metabolism. (b) Results obtained. The daily administration of eldecalcitol increased bone mass with the suppression of both bone resorption and bone formation. The suppression of bone resorption was more prominent than that of bone formation. Neither the number of QOPs nor the differentiation potential of QOPS into osteoclasts was affected by eldecalcitol administration. Eldecalcitol administration suppressed RANKL mRNA expression but not M-CSF or OPG mRNA expression in tibiae. Immunohistochemistry confirmed that the number of RANKL ${ }^{+}$cells in trabecular bones was decreased by eldecalcitol administration.

effectively inhibited bone resorption than calcitriol in vivo. ${ }^{62}$ These results suggest that the daily administration of active vitamin D compounds changes the bone microenvironments in osteoporotic patients.

\section{Why does the Daily Administration of Active Vitamin D Compounds Suppress Bone Resorption?}

There are several possible explanations for the suppression of bone resorption by active vitamin $\mathrm{D}$ compounds in vivo. The in vivo administration of active vitamin $D$ compounds may directly affect osteoblastic cells to suppress RANKL expression. However, this possibility is unlikely, because the suppression of RANKL expression by active vitamin D compounds has never been reported in vitro. The in vivo administration of large amounts of active vitamin D compounds to WT mice always induces osteoclastic bone resorption.

We believe that this phenomenon is induced by long-term exposure to pharmacological concentrations of active vitamin D compounds. Two potential mechanisms are conceivable for the in vivo reduction in RANKL expression in the bone (Figure 9). One possible mechanism is as follows: pharmacological concentrations of active vitamin D compounds in serum may alter the calcium endocrine system through intestine of parathyroid glands. Such an alteration in the calcium endocrine 
a

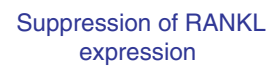

Active vitamin D compounds

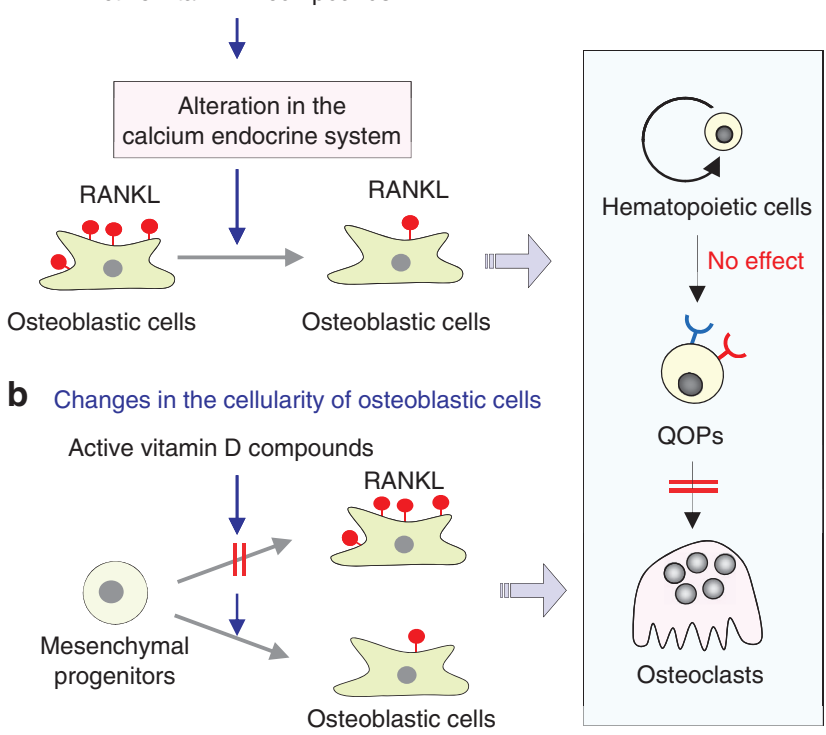

Figure 9 Two potential mechanisms for the in vivo effects of active vitamin $D$ compounds in inhibiting bone resorption. (a) Long-term exposure to active vitamin D compounds may influence the calcium endocrine system, which suppresses RANKL expression in osteoblastic cells in an integrated manner. (b) Daily administration of active vitamin D compounds may decrease RANKL activity in osteoblastic cells or induce a population shift of osteoblastic cells (changes in the cellularity of osteoblastic cells).

system may create favorable circumstances for the suppression of RANKL expression in osteoblastic cells (Figure 9a). Using intestinal-specific VDR ${ }^{-1-}$ mice, Lieben et al. ${ }^{63}$ showed that maintaining normocalcemic has priority over skeletal integrity. Daily administration of active vitamin D compounds may suppress bone resorption to maintain normocalcemia. Active vitamin $D$ compounds may also cause small but significant changes in the calcium endocrine system in vivo. Integrating such changes may suppress RANKL expression in osteoblastic cells (Figure 10).

The other possible mechanism is related to osteoblast differentiation: Daily administration of active vitamin D compounds may influence the differentiation of mesenchymal progenitors into osteoblastic cells, resulting in the suppression of RANKL expression (Figure 9b). RANKL is expressed by most osteoblast-lineage cells such as the bone marrow stromal cells, osteoblasts and osteocytes. RANKL was reported to be expressed preferentially by immature osteoblasts, and expression levels decreased during osteoblast maturation. ${ }^{64}$ Another study showed that osteocytes more effectively supported osteoclast formation in a co-culture than mature osteoblasts. ${ }^{65}$ de Freitas et al. ${ }^{66}$ reported that the daily administration of eldecalcitol to ovariectomized rats suppressed bone resorption and stimulated preosteoblasts to differentiate into mature osteoblasts in vivo. Gardiner et al. ${ }^{67}$ reported that transgenic mice overexpressing VDR in mature cells in the osteoblastic lineage showed increased bone formation and decreased bone resorption in mice. The transgene effects on bone formation and bone resorption exhibited site specificity of bone tissues. These results suggest that active vitamin $\mathrm{D}$ compounds directly act on osteoblastic cells to

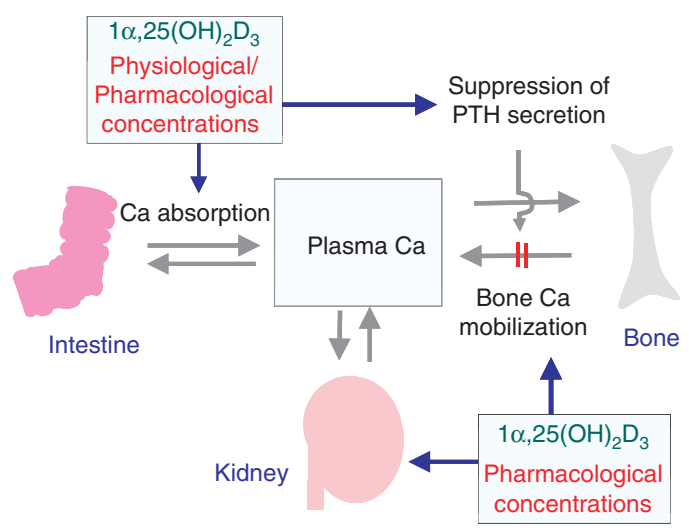

Figure 10 A hypothetical concept for the calcium endocrine system controlled by pharmacological concentrations of $1 \alpha, 25(\mathrm{OH})_{2} \mathrm{D}_{3} \cdot 1 \alpha, 25(\mathrm{OH})_{2} \mathrm{D}_{3}$ at physiological and pharmacological concentrations stimulates intestinal calcium absorption and inhibits PTH secretion. $1 \alpha, 25(\mathrm{OH})_{2} \mathrm{D}_{3}$ at pharmacological concentrations may inhibit PTH secretion and stimulate calcium renal calcium re-absorption. Integrating such significant changes may suppress RANKL expression in osteoblastic cells.

suppress bone resorption in a site-specific manner. These findings also suggest that active vitamin D compounds may decrease RANKL activity in osteoblastic cells on the trabecular bone surface or may induce a population shift of osteoblastic cells, resulting in a decrease in the number of RANKLexpressing osteoblasts.

The direct action of active vitamin $D$ compounds on osteoclast precursors has also been proposed to explain its suppressive effect on osteoclastogenesis. $1 \alpha, 25(\mathrm{OH})_{2} \mathrm{D}_{3}$ inhibits RANKL-induced osteoclastic differentiation in cultures of osteoclast precursors in the absence of osteoblastic cells. $1 \alpha, 25(\mathrm{OH})_{2} \mathrm{D}_{3}$ suppressed the expression of c-Fos, a transcription factor essential for osteoclastogenesis, in osteoclast precursors. ${ }^{68} 1 \alpha, 25(\mathrm{OH})_{2} \mathrm{D}_{3}$ was also shown to stimulate the expression of interferon $\beta$, an inhibitor of osteoclastogenesis, in osteoclast precursors. ${ }^{69}$ Kikuta et al. ${ }^{70}$ demonstrated that calcitriol and eldecalcitol inhibited bone resorption by modulating the S1P receptor system. The inhibitory effects of active vitamin $D$ compounds on osteoclast formation have been observed at concentrations higher than $10^{-9} \mathrm{M}$ in these in vitro experiments. Therefore, mechanisms other than the direct action of active vitamin $\mathrm{D}$ compounds on osteoclast precursors may be involved in active vitamin D-induced suppression of osteoclastogenesis in vivo.

\section{Conclusion}

Active vitamin $\mathrm{D}$ compounds stimulate osteoclast formation in a co-culture of osteoblastic cells and hematopoietic cells. Osteoblastic cells express RANKL in response to $1 \alpha, 25(\mathrm{OH})_{2} \mathrm{D}_{3}$. Therefore, $1 \alpha, 25(\mathrm{OH})_{2} \mathrm{D}_{3}$ has been believed to stimulate osteoclastic bone resorption. However, active vitamin D compounds are used as therapeutic drugs for osteoporosis, because they increase BMD in vivo due to the suppression of bone resorption. Thus, the effects of active vitamin D compounds on bone resorption in vitro and in vivo are opposite. We investigated the mechanism by which active vitamin D compounds inhibited bone resorption in vivo. QOPs were identified as the direct precursors of osteoclasts in vivo. Daily administration of eldecalcitol did not affect the generation of QOPs but 
suppressed RANKL expression in osteoblasts. Several possible explanations exist for the suppression of RANKL in osteoblasts by active vitamin $\mathrm{D}$ compounds in vivo. Pharmacological concentrations of active vitamin D compounds in serum may alter the calcium endocrine system, which may create circumstances for the suppression of RANKL expression in osteoblasts. An alternative possibility is the direct action of vitamin $\mathrm{D}$ on the bone: active vitamin $\mathrm{D}$ compounds may affect the cellularity of the osteoblast lineage. As a result, the number of RANKL-positive osteoblasts decreases. The direct action of active vitamin $\mathrm{D}$ compounds on osteoclast precursors has also been proposed to explain their suppressive effects on osteoclastogenesis. Further studies using osteoblast-specific VDR-deficient mice and also osteoclast precursor-specific VDR-deficient mice will elucidate the discrepancy observed between the in vitro and in vivo effects of active vitamin $\mathrm{D}$ compounds on bone resorption. Such experiments are currently being conducted in our laboratories.

\section{Conflict of Interest}

The authors declare no conflict of interest.

\section{Acknowledgements}

This work was supported by Grants-in-Aid for Science Research (22390351 and 22659339) from the Japan Society for the Promotion of Science. Parts of the present study were financially supported by Chugai Pharmaceutical Co., Ltd.

\section{References}

1. DeLuca HF. The vitamin D story: a collaborative effort of basic science and clinical medicine. FASEB J 1988;2:224-236.

2. Bouillon R, Okamura WH, Norman AW. Structure-function relationships in the vitamin $D$ endocrine system. Endocr Rev 1995;16:200-257.

3. McDonnell DP, Mangelsdorf DJ, Pike JW, Haussler MR, O'Malley BW. Molecular cloning of complementary DNA encoding the avian receptor for vitamin D. Science 1987;235:1214-1217.

4. Yoshizawa T, Handa Y, Uematsu Y, Takeda S, Sekine K, Yoshihara Y et al. Mice lacking the vitamin $\mathrm{D}$ receptor exhibit impaired bone formation, uterine hypoplasia and growth retardation after weaning. Nat Genet 1997;16:391-396.

5. Tanaka H, Seino $Y$. Direct action of 1,25-dihydroxyvitamin D on bone: VDRKO bone shows excessive bone formation in normal mineral condition. J Steroid Biochem Mol Biol 2004;89-90:343-345.

6. Carlsson A. Tracer experiments on the effect of vitamin $D$ on the skeletal metabolism of calcium and phosphorus. Acta Physiol Scand 1952;26:212-220.

7. Bauer GC. Bone salt metabolism in bone growth and bone repair studied in rats by means of $\mathrm{Ca}^{45}, \mathrm{P}^{32}$, and $\mathrm{Na}^{22}$. Acta Orthop Scand 1954;23:247-253.

8. Raisz LG, Trummel CL, Holick MF, DeLuca HF. 1,25-dihydroxycholecalciferol: a potent stimulator of bone resorption in tissue culture. Science 1972;175:768-769.

9. Roodman GD, Ibbotson KJ, MacDonald BR, Kuehl TJ, Mundy GR. 1,25-Dihydroxyvitamin $\mathrm{D}_{3}$ causes formation of multinucleated cells with several osteoclast characteristics in cultures of primate marrow. Proc Natl Acad Sci USA 1985;82:8213-8217.

10. Fuller K, Chambers TJ. Generation of osteoclasts in cultures of rabbit bone marrow and spleen cells. J Cell Physiol 1987;132:441-452.

11. Takahashi N, Yamana H, Yoshiki S, Roodman GD, Mundy GR, Jones SJ et al. Osteoclast-like cell formation and its regulation by osteotropic hormones in mouse bone marrow cultures. Endocrinology 1988;122:1373-1382.

12. Tilyard MW, Spears GF, Thomson J, Dovey S. Treatment of postmenopausal osteoporosis with calcitriol or calcium. N Engl J Med 1992;326:357-362.

13. Orimo H, Shiraki M, Hayashi $\mathrm{Y}$, Hoshino T, Onaya T, Miyazaki S et al. Effects of $1 \alpha$ hydroxyvitamin $\mathrm{D}_{3}$ on lumbar bone mineral density and vertebral fractures in patients with postmenopausal osteoporosis. Calcif Tissue Int 1994;54:370-376.

14. Matsumoto T, Miki T, Hagino H, Sugimoto T, Okamoto S, Hirota T et al. A new active vitamin D, ED-71, increases bone mass in osteoporotic patients under vitamin D supplementation: a randomized, double-blind, placebo-controlled clinical trial. J Clin Endocrinol Metab 2005:90:5031-5036.

15. Matsumoto T, Takano T, Yamakido S, Takahashi F, Tsuji N. Comparison of the effects of eldecalcitol and alfacalcidol on bone and calcium metabolism. J Steroid Biochem Mol Biol 2010;121:261-264.
16. Matsumoto $T$, Ito $M$, Hayashi $Y$, Hirota $T$, Tanigawara $Y$, Sone $T$ et al. A new active vitamin $D_{3}$ analog, eldecalcitol, prevents the risk of osteoporotic fractures-a randomized, active comparator, double-blind study. Bone 2011;49:605-612.

17. Nakamura I, Takahashi N, Jimi E, Udagawa N, Suda T. Regulation of osteoclast function. Mod Rheumatol 2012;22:167-177.

18. Vaananen HK, Laitala-Leinonen T. Osteoclast lineage and function. Arch Biochem Biophys 2008:473:132-138.

19. Fuller K, Ross JL, Szewczyk KA, Moss R, Chambers TJ. Bone is not essential for osteoclast activation. PLoS One 2010;5: pii: e12837.

20. Kukita T, Wada N, Kukita A, Kakimoto T, Sandra F, Toh K et al. RANKL-induced DC-STAMP is essential for osteoclastogenesis. J Exp Med 2004;200:941-946.

21. Yagi M, Miyamoto T, Sawatani $\mathrm{Y}$, Iwamoto K, Hosogane N, Fujita $\mathrm{N}$ et al. DC-STAMP is essential for cell-cell fusion in osteoclasts and foreign body giant cells. J Exp Med 2005;202:345-351.

22. Miyamoto H, Suzuki T, Miyauchi Y, Iwasaki R, Kobayashi T, Sato Y et al. Osteoclast stimulatory transmembrane protein and dendritic cell-specific transmembrane protein cooperatively modulate cell-cell fusion to form osteoclasts and foreign body giant cells. J Bone Miner Res 2012;27:1289-1297.

23. Rodan GA, Martin TJ. Role of osteoblasts in hormonal control of bone resorption-a hypothesis. Calcif Tissue Int 1981;33:349-351.

24. Chambers TJ. The cellular basis of bone resorption. Clin Orthop Relat Res 1980;151:283-293.

25. Takahashi N, Akatsu T, Udagawa N, Sasaki T, Yamaguchi A, Moseley JM et al. Osteoblastic cells are involved in osteoclast formation. Endocrinology 1988;123:2600-2602.

26. Yoshida H, Hayashi S, Kunisada T, Ogawa M, Nishikawa S, Okamura $\mathrm{H}$ et al. The murine mutation osteopetrosis is in the coding region of the macrophage colony stimulating factor gene. Nature 1990;345:442-444.

27. Felix R, Cecchini MG, Fleisch $\mathrm{H}$. Macrophage colony stimulating factor restores in vivo bone resorption in the op/op osteopetrotic mouse. Endocrinology 1990;127:2592-2594.

28. Takahashi N, Udagawa N, Akatsu T, Tanaka H, Isogai Y, Suda T. Deficiency of osteoclasts in osteopetrotic mice is due to a defect in the local microenvironment provided by osteoblastic cells. Endocrinology 1991;128:1792-1796.

29. Tanaka S, Takahashi N, Udagawa N, Tamura T, Akatsu T, Stanley ER et al. Macrophage colony-stimulating factor is indispensable for both proliferation and differentiation of osteoclast progenitors. J Clin Invest 1993;91:257-263.

30. Suda T, Takahashi N, Martin TJ. Modulation of osteoclast differentiation. Endocr Rev 1992:13:66-80

31. Chambers TJ, Owens JM, Hattersley G, Jat PS, Noble MD. Generation of osteoclast-inductive and osteoclastogenic cell lines from the H-2KbtsA58 transgenic mouse. Proc Natl Acad Sci USA 1993;90:5578-5582.

32. Simonet WS, Lacey DL, Dunstan CR, Kelley M, Chang MS, Luthy R et al. Osteoprotegerin: a novel secreted protein involved in the regulation of bone density. Cell 1997;89:309-319.

33. Yasuda H, Shima N, Nakagawa N, Mochizuki SI, Yano K, Fujise N et al. Identity of osteoclastogenesis inhibitory factor (OCIF) and osteoprotegerin (OPG): a mechanism by which OPG/OCIF inhibits osteoclastogenesis in vitro. Endocrinology 1998;139:1329-1337.

34. Yasuda H, Shima N, Nakagawa N, Yamaguchi K, Kinosaki M, Mochizuki S et al. Osteoclast differentiation factor is a ligand for osteoprotegerin/osteoclastogenesis-inhibitory factor and is identical to TRANCE/RANKL. Proc Natl Acad Sci USA 1998;95:3597-3602.

35. Lacey DL, Timms E, Tan HL, Kelley MJ, Dunstan CR, Burgess T et al. Osteoprotegerin ligand is a cytokine that regulates osteoclast differentiation and activation. Cell 1998;93: 165-176.

36. Wong BR, Rho J, Arron J, Robinson E, Orlinick J, Chao M et al. TRANCE is a novel ligand of the tumor necrosis factor receptor family that activates c-Jun N-terminal kinase in T cells. J Biol Chem 1997;272:25190-25194.

37. Anderson DM, Maraskovsky E, Billingsley WL, Dougall WC, Tometsko ME, Roux ER et al. A homologue of the TNF receptor and its ligand enhance T-cell growth and dendritic-cell function. Nature 1997;390:175-179.

38. Suda T, Takahashi N, Udagawa N, Jimi E, Gillespie MT, Martin TJ. Modulation of osteoclast differentiation and function by the new members of the tumor necrosis factor receptor and ligand families. Endocr Rev 1999;20:345-357.

39. Boyle WJ, Simonet WS, Lacey DL. Osteoclast differentiation and activation. Nature 2003:423:337-342.

40. Takeda S, Yoshizawa T, Nagai Y, Yamato H, Fukumoto S, Sekine K et al. Stimulation of osteoclast formation by 1,25 -dihydroxyvitamin $D$ requires its binding to vitamin $D$ receptor (VDR) in osteoblastic cells: studies using VDR knockout mice. Endocrinology 1999;140: 1005-1008.

41. Pike JW, Lee SM, Meyer MB. Regulation of gene expression by 1,25-dihydroxyvitamin $D_{3}$ in bone cells: exploiting new approaches and defining new mechanisms. BoneKEy Reports 3 , Article number: 482 (2014); doi:10.1038/bonekey.2013.216.

42. Takayanagi H. Osteoimmunology: shared mechanisms and crosstalk between the immune and bone systems. Nat Rev Immunol 2007;7:292-304.

43. Kuroda Y, Matsuo K. Molecular mechanisms of triggering, amplifying and targeting RANK signaling in osteoclasts. World J Orthop 2012;3:167-174.

44. Takayanagi $\mathrm{H}$, Kim S, Koga $\mathrm{T}$, Nishina $\mathrm{H}$, Isshiki M, Yoshida $\mathrm{H}$ et al. NFATc1 (NFAT2) integrate RANKL signaling in terminal differentiation of osteoclasts. Dev Cell 2002;3: 889-901.

45. Koga T, Inui M, Inoue K, Kim S, Suematsu A, Kobayashi E et al. Costimulatory signals mediated by the ITAM motif cooperate with RANKL for bone homeostasis. Nature 2004:428:758-763. 
46. Barrow AD, Raynal N, Andersen TL, Slatter DA, Bihan D, Pugh N et al. OSCAR is a collagen receptor that costimulates osteoclastogenesis in DAP12-deficient humans and mice. J Clin Invest 2011;121:3505-3516.

47. Kuroda Y, Hisatsune C, Nakamura T, Matsuo K, Mikoshiba K. Osteoblasts induce $\mathrm{Ca}^{2+}$ oscillation-independent NFATc1 activation during osteoclastogenesis. Proc Natl Acad Sci USA 2008:105:8643-8648.

48. Kuroda Y, Hisatsune C, Mizutani A, Ogawa N, Matsuo K, Mikoshiba K. Cot kinase promotes $\mathrm{Ca} 2+$ oscillation/calcineurin-independent osteoclastogenesis by stabilizing NFATc1 protein. Mol Cell Biol 2012;32:2954-2963.

49. Mizoguchi T, Muto A, Udagawa N, Arai A, Yamashita T, Hosoya A et al. Identification of cell cycle-arrested quiescent osteoclast precursors in vivo. J Cell Biol 2009;184:541-554.

50. Muto A, Mizoguchi T, Udagawa N, Ito S, Kawahara I, Abiko $Y$ et al. Lineage-committed osteoclast precursors circulate in blood and settle down into bone. J Bone Miner Res 2011;26:2978-2990.

51. Yamamoto Y, Udagawa N, Matsuura S, Nakamichi Y, Horiuchi H, Hosoya A et al. Osteoblasts provide a suitable microenvironment for the action of receptor activator of nuclear factorkappaB ligand. Endocrinology 2006:147:3366-3374.

52. Kotani M, Kikuta J, Klauschen F, Chino T, Kobayashi Y, Yasuda $\mathrm{H}$ et al. Systemic circulation and bone recruitment of osteoclast precursors tracked by using fluorescent imaging techniques. J Immunol 2013;190:605-612.

53. Lin H, Lee E, Hestir K, Leo C, Huang M, Bosch E et al. Discovery of a cytokine and its receptor by functional screening of the extracellular proteome. Science 2008:320:807-811.

54. Nakamichi Y, Mizoguchi T, Arai A, Kobayashi Y, Sato M, Penninger JM et al. Spleen serves as a reservoir of osteoclast precursors through vitamin D-induced IL-34 expression in osteopetrotic op/op mice. Proc Natl Acad Sci USA 2012;109:10006-10011.

55. Arai F, Miyamoto T, Ohneda O, Inada T, Sudo T, Brasel K et al. Commitment and differentiation of osteoclast precursor cells by the sequential expression of $\mathrm{c}-\mathrm{Fms}$ and receptor activator of nuclear factor kappaB (RANK) receptors. J Exp Med 1999;190:1741-1754.

56. Arai A, Mizoguchi T, Harada S, Kobayashi Y, Nakamichi $\mathrm{Y}$, Yasuda $\mathrm{H}$ et al. c-Fos plays an essential role in the up-regulation of RANK expression in osteoclast precursors within the bone microenvironment. J Cell Sci 2012;125:2910-2917.

57. Ishii M, Egen JG, Klauschen F, Meier-Schellersheim M, Saeki Y, Vacher J et al. Sphingosine-1phosphate mobilizes osteoclast precursors and regulates bone homeostasis. Nature 2009;458:524-528.

58. Morimoto S, Imanaka S, Koh E, Shiraishi T, Nabata T, Kitano S et al. Comparison of the inhibitions of proliferation of normal and psoriatic fibroblasts by $1 \alpha, 25$-dihydroxyvitamin D3 and synthetic analogues of vitamin D3 with an oxygen atom in their side chain. Biochem Int 1989;19:1143-1149.

59. Okano T, Tsugawa N, Masuda S, Takeuchi A, Kobayashi T, Nishii Y. A novel synthetic vitamin $\mathrm{D}_{3}$ analogue, 2 $\beta$-(3-hydroxypropoxy)-calcitriol (ED-71): its biological activities and pharmacological effects on calcium metabolism. Contrib Nephrol 1991;91:116-122.
60. Okano T, Tsugawa N, Masuda S, Takeuchi A, Kobayashi T, Takita Y et al. Regulatory activities of $2 \beta$-(3-hydroxypropoxy)-1 $\alpha$, 25-dihydroxyvitamin $D_{3}$, a novel synthetic vitamin $D_{3}$ derivative, on calcium metabolism. Biochem Biophys Res Commun 1989;163: 1444-1449.

61. Morris HA, Anderson PH. Autocrine and paracrine actions of vitamin D. Clin Biochem Rev 2010;31:129-138.

62. Harada S, Mizoguchi T, Kobayashi Y, Nakamichi Y, Takeda S, Sakai S et al. Daily administration of eldecalcitol (ED-71), an active vitamin $D$ analog, increases bone mineral density by suppressing RANKL expression in mouse trabecular bone. $J$ Bone Miner Res 2012;27:461-473.

63. Lieben L, Masuyama R, Torrekens S, Van Looveren R, Schrooten J, Baatsen P et al. Normocalcemia is maintained in mice under conditions of calcium malabsorption by vitamin D-induced inhibition of bone mineralization. J Clin Invest 2012;122: $1803-1815$.

64. Atkins GJ, Kostakis P, Pan B, Farrugia A, Gronthos S, Evdokiou A et al. RANKL expression is related to the differentiation state of human osteoblasts. $J$ Bone Miner Res 2003;18: 1088-1098.

65. Nakashima T, Hayashi M, Fukunaga T, Kurata K, Oh-Hora M, Feng JQ et al. Evidence for osteocyte regulation of bone homeostasis through RANKL expression. Nat Med 2011:17:1231-1234.

66. de Freitas PH, Hasegawa T, Takeda S, Sasaki M, Tabata C, Oda K et al. Eldecalcitol, a secondgeneration vitamin $D$ analog, drives bone minimodeling and reduces osteoclastic number in trabecular bone of ovariectomized rats. Bone 2011;49:335-342.

67. Gardiner EM, Baldock PA, Thomas GP, Sims NA, Henderson NK, Hollis B et al. Increased formation and decreased resorption of bone in mice with elevated vitamin $D$ receptor in mature cells of the osteoblastic lineage. FASEB J 2000;14:1908-1916.

68. Takasu H, Sugita A, Uchiyama Y, Katagiri N, Okazaki M, Ogata E et al. c-Fos protein as a target of anti-osteoclastogenic action of vitamin $\mathrm{D}$, and synthesis of new analogs. $J$ Clin Invest 2006;116:528-535.

69. Sakai S, Takaishi H, Matsuzaki K, Kaneko H, Furukawa M, Miyauchi $Y$ et al. 1-Alpha 25-dihydroxy vitamin $D_{3}$ inhibits osteoclastogenesis through IFN-beta-dependent NFATc1 suppression. J Bone Miner Metab 2009;27:643-652.

70. Kikuta J, Kawamura S, Okiji F, Shirazaki M, Sakai S, Saito H et al. Sphingosine-1phosphate-mediated osteoclast precursor monocyte migration is a critical point of control in antibone-resorptive action of active vitamin D. Proc Natl Acad Sci USA 2013;110:7009-7013.

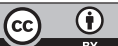

This work is licensed under a Creative Commons Attribution 3.0 Unported License. To view a copy of this license, visit http:// creativecommons.org/licenses/by/3.0/ 